\title{
Improved Radio Frequency Identification Indoor Localization Method via Radial Basis Function Neural Network
}

\author{
Dongliang Guo, ${ }^{1}$ Yudong Zhang, ${ }^{2,3}$ Qiao Xiang, ${ }^{1}$ and Zhonghua Li ${ }^{1}$ \\ ${ }^{1}$ School of Information Science and Technology, Sun Yat-sen University, Guangzhou 510006, China \\ ${ }^{2}$ School of Information Science and Technology, Nanjing Normal University, Nanjing 210023, China \\ ${ }^{3}$ MRI Unit, Columbia University and New York State Psychiatric Institute, New York, NY 10032, USA \\ Correspondence should be addressed to Zhonghua Li; lizhongh@mail.sysu.edu.cn
}

Received 3 January 2014; Revised 10 June 2014; Accepted 15 June 2014; Published 10 July 2014

Academic Editor: Ezzat G. Bakhoum

Copyright (C) 2014 Dongliang Guo et al. This is an open access article distributed under the Creative Commons Attribution License, which permits unrestricted use, distribution, and reproduction in any medium, provided the original work is properly cited.

Indoor localization technique has received much attention in recent years. Many techniques have been developed to solve the problem. Among the recent proposed methods, radio frequency identification (RFID) indoor localization technology has the advantages of low-cost, noncontact, non-line-of-sight, and high precision. This paper proposed two radial basis function (RBF) neural network based indoor localization methods. The RBF neural networks are trained to learn the mapping relationship between received signal strength indication values and position of objects. Traditional method used the received signal strength directly as the input of neural network; we added another input channel by taking the difference of the received signal strength, thus improving the reliability and precision of positioning. Fuzzy clustering is used to determine the center of radial basis function. In order to reduce the impact of signal fading due to non-line-of-sight and multipath transmission in indoor environment, we improved the Gaussian filter to process received signal strength values. The experimental results show that the proposed method outperforms the existing methods as well as improves the reliability and precision of the RFID indoor positioning system.

\section{Introduction}

With the rapid development of data services and multimedia services, the demand for positioning and navigation is increasing day by day. In the complex indoor environment, it is needed to determine the location of facilities or personnel. Indoor localization technique has caused widespread concern. Indoor localization facilitates applications such as detection of products in workshop or warehouse, detection of medical personnel or equipment in a hospital, and autonomous robot localization.

In outdoor environment, the satellite-based positioning techniques, such as the global positioning system (GPS), have been widely applied. However, the signal of satellitebased positioning system is incapable to penetrate into buildings. To solve this problem, indoor localization technologies are necessary. Existing technologies include the wireless local area networks (WLAN), ultrasonic positioning, infrared, and radio frequency identification (RFID) [1]. However, almost every indoor localization system has some disadvantages. For instance, infrared is very weak in penetrability and the distance it can transmit is too short, ultrasonic positioning apparatus is too expensive, and WLAN cannot provide high accuracy. Compared to other technologies, RFID is particularly suitable for indoor localization. RFID has the advantages of low-cost, noncontact, non-line-of-sight, and high precision, which is a preferable technology for indoor localization [2].

There are several kinds of approaches in RFID-based indoor localization system. Some approaches depend on timing measurements, such as measuring time of arrival (TOA) [3] or time difference of arrival (TDOA) [4], some approaches depend on measuring angle of arrival (AOA) [5]. However, the most popular methods rely on measuring the received signal strength Indication (RSSI) [6]. Exploiting RSSI value for positioning is the main method of indoor localization.

In this study, we present two improved radial basis function (RBF) neural network based RFID indoor positioning approach utilizing RSSI parameter. The major advantages of the RBF neural network over other types of neural networks include simple network structure, fast learning, and good approximation ability [7]. 
Usually, the received signal strength is directly used as the input of neural network [8-15]. Different from the usual methods, we improved the input method by taking the difference of the received signal strength as another input channel, thus improving the reliability and precision of positioning. Fuzzy clustering is used to determine the center of radial basis function. In order to reduce the impact of signal fading due to non-line-of-sight and multipath transmission in indoor environment, we improved the Gaussian filter to process received signal strength values. Two technical schemes were proposed; experimental results and technical discussion were given in this paper.

The rest of the paper is organized as follows. Section 2 describes related works in the field of artificial neural networks (ANNs) based RFID indoor positioning. The proposed RBF neural network based RFID indoor localization methods are given in Section 3. The comparison between the derived methods and existing solutions is presented in Section 4. Conclusions and discussion are given in Section 5.

\section{Review of Related Theories}

2.1. RFID Indoor Localization. RFID is a noncontact and nonline-of-sight technology for detecting the monitored objects configuring a RFID tag in a given area. RFID-based location system is the preferred technology with its low-cost, noncontact, non-line-of-sight, and high precision [2]. A RFID system is composed of RFID readers and RFID tags. RFID readers communicate with the tags in its detectable scope and transmit the information received from tags to a host computer. RFID tag is an integrated circuit with antenna. Each RFID tag has a unique identifier (ID). RFID tags could be categorized into active and passive tags. Active tag is powered by its own battery, and therefore it could be detected in a larger distance. Passive RFID tags operate without a battery, and it receives and responses to RF signal from a RFID reader. With the improvement of technology and its characteristics, RFID has been successfully applied to indoor positioning.

RFID indoor localization techniques can be divided into three categories regarding the methodology used: triangulation, fingerprinting based, and proximity [1]. Among them, fingerprinting based methods are the most commonly used techniques. They are carried out in two phases: the offline and the online phase. The offline phase comprises collecting the RSSI values from RFID readers and storing them, along with the position information of the tags, into a database. In the online phase, the object's position is estimated by comparing the likeliness of the RSSI vector measured during the online phase with the previously stored RSSI vectors in the database. Fingerprinting based techniques are important and have yielded good performance.

2.2. Neural Networks Based RFID Indoor Localization. The artificial neural networks (ANNs) based RFID indoor localization methods are classified as fingerprinting technique. $\mathrm{ANN}$ is a signal process and pattern recognition tool that can still yield good results with noisy signals. In the offline phase, the set of collected RSSI vectors is used to train the network and tune its inner coefficients/weights. In the online phase,

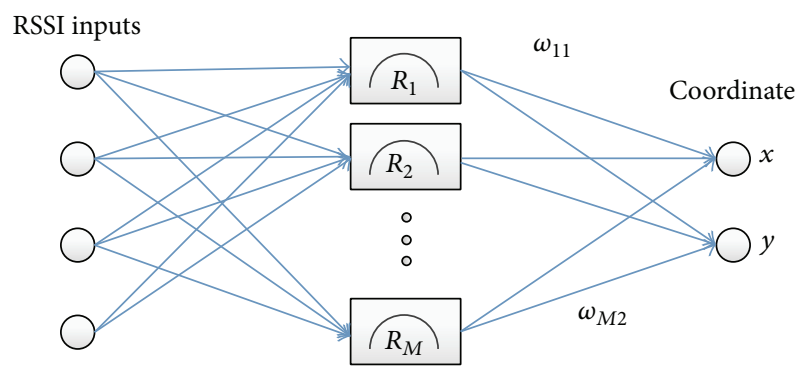

FIGURE 1: Structure of the RBF neural network.

the trained neural network performs localization processes. Utilizing ANNs requires no detailed knowledge of the RFID readers' positions or the indoor environment. Although training is time-consuming, the prediction or classification process is much faster than analytical method. ANNs based RFID indoor localization method is the current research focus, while many related algorithms were developed and explored.

Back-Propagation (BP) neural networks were used to perform indoor positioning, including a single $\mathrm{BP}$ network and several BP networks [9-11]; the result shows that the BP neural networks can solve the problems caused by the complication and variation of indoor environment at a certain degree.

Fuzzy neural network architecture is developed to accomplish indoor positioning and active tag is used in the proposed system $[12,13]$. The advantage of fuzzy neural network is its self-learning ability, which is utilized to indoor targets positioning.

Radial basis function neural network (RBFNN) is developed to accomplish indoor positioning $[14,15]$. In [14], a RBFNN trained via a minimization of the localized generalization error is adopted to learn the object location based on received RSSI values. In [15], a hybrid RFID/IR indoor localization mechanism applied to smart buildings is proposed, a $\mathrm{RBF}$ network is used to estimate the location of occupants, and a particle filter is used to track their next positions.

The advantages of RBFNN over other types of ANNs include simple network structure, fast learning, and good approximation ability, so we choose RBFNN to realize RFID indoor positioning. RBFNN consists of an input layer, a hidden layer, and an output layer. A RBFNN is defined by the number of hidden neurons, center vector and width value used to define the Gaussian activation of each hidden neuron, and the connection weights between hidden neurons and the output neurons, as shown in Figure 1. The RSSI vector is imported into the input layer; the number of neurons in hidden layer is $M$ and the number of neurons in output layer is equal to 2, corresponding to the estimated coordinate $(x, y)$.

\section{Improved RFID Indoor Localization Method via RBFNN}

3.1. Utilizing the Difference of RSSI Values. In general, if there are $n$ RFID readers in the indoor localization system, the 


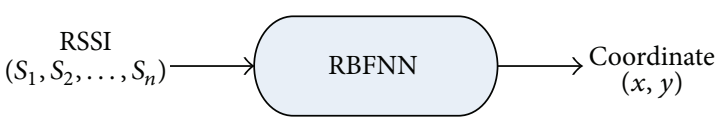

(a) The general scheme

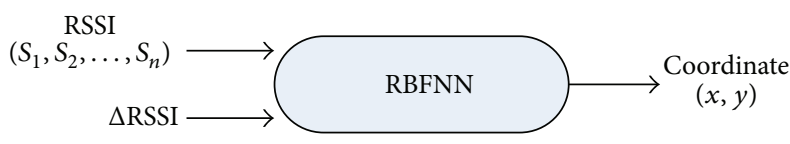

(b) The proposed scheme

FIGURE 2: Artificial neural network based indoor positioning system.

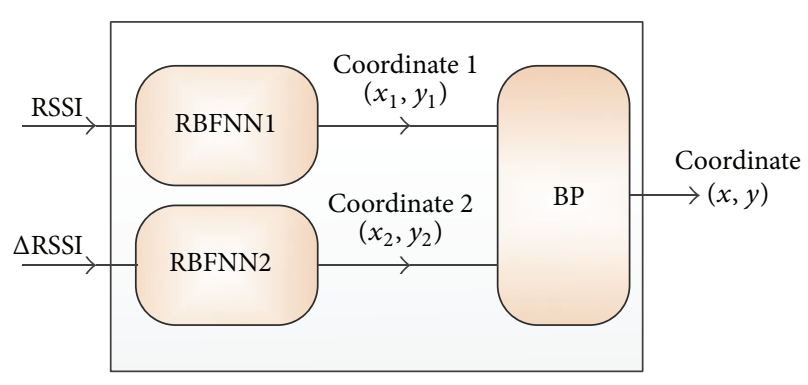

(a) Method I: separate RBF neural networks

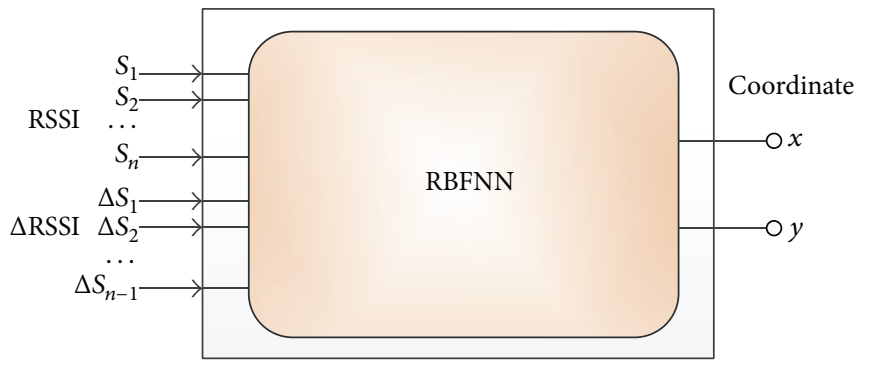

(b) Method II: integrate RBF neural network

FIGURE 3: Proposed RBF neural network structure.

input layer of RBFNN should include $n$ nodes corresponding to the $n$ RSSI values $\left(S_{1}, S_{2}, \ldots, S_{n}\right)$, as shown in Figure 2(a). The output layer has two nodes corresponding to the coordinate $(x, y)$.

In our work, we found that input the differential information of received signal strength $\triangle$ RSSI drawn from the original RSSI value $\left(S_{1}, S_{2}, \ldots, S_{n}\right)$ can enhance the reliability and precision of positioning, the proposed scheme is shown in Figure 2(b).

If the RSSI value is $\left(S_{1}, S_{2}, \ldots, S_{n}\right)$, we can calculate the difference information as follows:

$$
\begin{gathered}
\Delta S_{1}=S_{1}-S_{2}, \\
\Delta S_{2}=S_{1}-S_{3}, \\
\vdots \\
\Delta S_{n-1}=S_{1}-S_{n} .
\end{gathered}
$$

In addition, we name the difference information as $\Delta$ RSSI = $\left(\Delta S_{1}, \Delta S_{2}, \ldots \Delta S_{n-1}\right)$.

Using the RSSI value to perform indoor localization computing is equivalent to using $n$ circle to compute the intersection point in triangulation method, the radius of the circles is the distance calculated from the RSSI value through the signal propagation model and the centers are the RFID readers. Using the difference information $\Delta$ RSSI together with RSSI is equivalent to using TOA method together with TDOA method. In the ideal situation of no RSSI fluctuation, one method can complete the positioning accurately, but in the presence of signal fluctuation, using two methods together can increase the positioning reliability and accuracy. Based on this idea, we propose two RBFNN based indoor localization methods, as shown in Figure 3.

Method I is shown in Figure 3(a); in this method, RSSI and $\triangle$ RSSI were input to RBFNN1 and RBFNN2 separately, and the outputs of RBFNN1 and RBFNN2 were input to a BP neural network, the output of BP network is the coordinate $(x, y)$ of the tracked tag. Method II is shown in Figure 3(b); in this method, RSSI and $\Delta$ RSSI were input to an integrate $\mathrm{RBF}$ neural network, the output is the coordinate $(x, y)$.

3.2. Processing and Calculation of the Received RSSI. In the actual indoor environment, the RSSI values received by the readers fluctuate greatly as a consequence of non-line-ofsight and multipath transmission, which leads to the deviation of positioning calculation. In order to reduce the impact of signal fading, we improved the Gaussian filter [16] to process the received RSSI values.

In theory, the relationship of the RSSI value and the distance from a reader to a tag satisfies indoor path-loss model [17] as

$$
\begin{aligned}
\operatorname{RSSI}(d)[\mathrm{dBm}]= & P_{T}[\mathrm{dBm}]-\operatorname{PL}\left(d_{0}\right)[\mathrm{dB}] \\
& -10 \gamma \log _{10}\left(\frac{d}{d_{0}}\right)[\mathrm{dB}]+X_{\sigma}(\mathrm{dB}),
\end{aligned}
$$

where $\operatorname{RSSI}(d)$ is the RSSI value at the transmitter-receiver separation distance $d, P_{T}$ is the power of transmit signal, $\gamma$ is the path loss exponent which indicates the rate of path loss with respect to distance, $\operatorname{PL}(\cdot)$ is path loss and $d_{0}$ is the distance between the transmitter and the reference point, and $X_{\sigma}$ is a zero mean log-normally distributed random variable.

The density function of RSSI values is shown as

$$
f(\mathrm{RSSI})=\frac{1}{\sqrt{2 \pi} \sigma} \exp \left[-\frac{(\mathrm{RSSI}-A)^{2}}{2 \sigma^{2}}\right],
$$

where $\sigma^{2}$ is the variance and $A$ is the expected value.

We improve the original Gaussian filter to adapt the filtering of RSSI value, first we estimate the standard deviation via

$$
\widehat{\sigma}=\sqrt{\frac{1}{n-1} \sum_{i=1}^{n}\left(\mathrm{RSSI}_{i}-\widehat{A}\right)^{2}}
$$


where $\widehat{A}$ is the estimation of expected value of RSSI, as

$$
\widehat{A}=\frac{1}{n} \sum_{i=1}^{n} \operatorname{RSSI}_{i} .
$$

The output value of Gaussian filter is the arithmetic mean of the RSSI values in the numerical interval $[\widehat{A}-\widehat{\sigma}, \widehat{A}+\widehat{\sigma}]$, as

$$
\mathrm{RSSI}_{\mathrm{opt}}=\frac{1}{L} \sum_{j=1}^{L} \operatorname{RSSI}_{j} .
$$

3.3. The Proposed Methodology. The proposed method is a supervised learning method that learns the relationship between RSSI values and position of RFID reference tags. The RBFNN outputs the estimation of the coordinate of the tagged object as $(x, y)$. The process of the proposed method is as follows.

(1) Deploy RFID Readers and Reference Tags. Suppose there are $n$ readers, $m$ reference tags, and $u$ tracking tags in the positioning area. $n$ readers are installed on the ceiling of the building to cover the positioning regions. $m$ reference tags are deployed in the regions for location identification. A RFID tag is used for each object being tracked.

(2) Collect Training Samples. For each reference tag, there will be $n$ readers' RSSI values $\operatorname{RSSI}_{i}=\left(S_{i 1}, S_{i 2}, \ldots, S_{i n}\right), i=1 \sim$ $m$, indicating the relative location of the reference tags with respect to those readers.

The RSSI values of tags are first Gaussian filtered to reduce the impact of signal fading. We use the improved method mentioned in Section 3.2 to calculate the signal strength. This preprocess will improve the positioning accuracy. We then construct a training dataset with $m$ training samples.

(3) Train Radial Basis Function Neural Network (RBFNN). Method I: Two independent RBFNN are trained; RBFNN1 is trained using RSSI vector and RBFNN2 is trained using $\triangle \mathrm{RSSI}$ vector, the outputs of RBFNN1 and RBFNN2 are used to train a BP neural network, the tutor is the coordinate of the reference tags.

Method II: An integrate RBFNN is trained using RSSI and $\Delta$ RSSI.

The most widely adopted RBFNN training procedures are divided into two phases: unsupervised and supervised. In the unsupervised phase, with a fixed $M$ value, centers are allocated by a clustering of the training samples into $M$ clusters and their centroids are used as the center vectors for hidden neurons of the RBFNN. Once the number of hidden neurons is fixed, the RBFNN architecture will be determined. The number of hidden neurons has a great influence on the generalization capability of a RBFNN. If the number of hidden neurons is too small, the RBFNN could not learn well from the training samples. On the other hand, if the number of hidden neurons is too large, the RBFNN will overfit [18]. Therefore, the optimal number of hidden neurons should be selected for a RBFNN to achieve a good generalization capability. In our work, a kind of fuzzy clustering algorithm is adopted to determine the center of radial basis function. The width of hidden neuron is computed by the average distances among all centroids. In the supervised learning phase, the connection weights are computed via the pseudo inverse method.

The optimal number $M^{*}$ of hidden neurons for a RBFNN is selected as follows.

(1) Start from $M=2$;

(2) fuzzy clustering to get $M$ centers, train a RBFNN with $M$ hidden neurons until the network converges and then compute the network's mean positioning error;

(3) if $M<m, M \leftarrow M+1$ and go to step 2;

(4) the number of hidden neurons $M^{*}$ yielding the minimum positioning error is selected.

(4) Indoor Localization and Re-Train the Network. When the tracked object configuring a RFID tag move into the localization region, the localization system will gets the signal strength of the tracked tag, after improved Gaussian filtering and calculation, we get RSSI and $\Delta$ RSSI, these data is input to the neural network to calculate object location.

The major challenge of indoor positioning is that the indoor environment will change occasionally. When there is change of indoor environment, we could retrain the network using those reference tags with known location. This adaptive learning helps the system to adapt changes in the indoor environment. The time period between retraining could be selected by user based on the changes of the indoor environment.

Figure 4 shows the processes of the proposed RBFNN based indoor localization methods.

\section{Experiments and Results}

In this section, we design several numerical experiments to examine the performance of the proposed methods for indoor localization and to make some comparisons with other algorithms. Besides, some technical details, simulated experimental results and corresponding discussions are given.

4.1. Experimental Environment and Parameter Setting. Numerical experiments are performed in a $10 \mathrm{~m}$ by $10 \mathrm{~m}$ room. Four readers are used to cover the room. Tracked tags are placed in the same space randomly to evaluate the localization performance.

Given the RSSI vector $\left(S_{1}, S_{2}, S_{3}, S_{4}\right)$, we set the difference information of $\Delta$ RSSI as $\Delta$ RSSI $=\left(\Delta S_{1}, \Delta S_{2}, \Delta S_{3}\right)$, where

$$
\begin{aligned}
& \Delta S_{1}=S_{1}-S_{2}, \\
& \Delta S_{2}=S_{1}-S_{3}, \\
& \Delta S_{3}=S_{1}-S_{4} .
\end{aligned}
$$

In method I, RBFNN1 has 4 neurons in the input layer and RBFNN2 has 3 neurons in the input layer. The output layer of RBFNN1 or RBFNN2 has 2 neurons, respectively. There are 4 


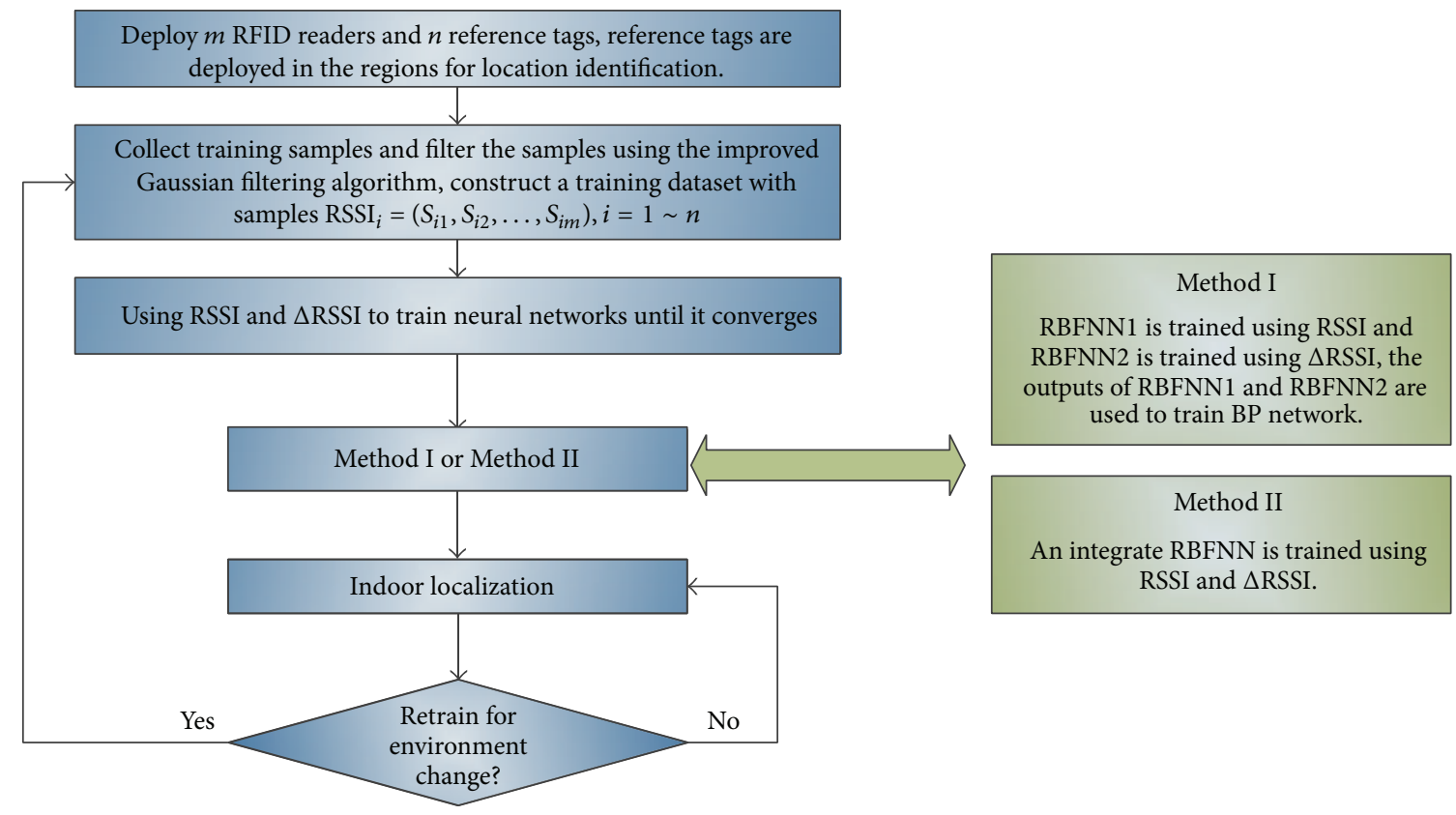

FIGURE 4: The processes of the proposed RBFNN based indoor localization methods.

neurons in the input layer and 2 neurons in the output layer of the BP network. In method II, the integrated RBFNN has 7 neurons in the input layer and 2 neurons in the output layer.

The positioning error is determined by the Euclidean distance between the estimated location and the real location of the tagged object, as

$$
\text { error }=\sqrt{\left(x-x_{0}\right)^{2}+\left(y-y_{0}\right)^{2}}
$$

where $\left(x_{0}, y_{0}\right)$ is the real location of the tagged object and $(x, y)$ is the estimated location of the tagged object.

Simulation data is created by a signal loss model in [18] as follows:

$$
\mathrm{PL}(d)[\mathrm{dB}]=\mathrm{PL}\left(d_{0}\right)[\mathrm{dB}]+10 \gamma \log _{10}\left(\frac{d}{d_{0}}\right)+X_{\sigma},
$$

where $d_{0}, \operatorname{PL}\left(d_{0}\right), \operatorname{PL}(d), \gamma$, and $X_{\sigma}$ denote reference distance between reader and reference point, signal loss from reader to reference point, and signal loss from reader to the point with a distance of $d$, the mean path loss exponent, and the fading in environment, respectively.

Parameters $\gamma$ and $X_{\sigma}$ are determined by positioning environment. $X_{\sigma}$ is a zero mean log-normally distributed random variable. Parameters used in our simulation are listed in Table 1.

4.2. Experimental Results. The coordinates of readers are $(2.7,3.3),(2.3,7.7),(7.7,2.5)$, and $(7.3,6.7)$, respectively. The distances among neighboring reference tags are horizontally and vertically set to be $1 \mathrm{~m}$, respectively. In order to distinguish with later experiments, we named this experimental condition as Case 0 . The deployment of readers and reference tags of Case 0 is shown in Figure 5.
TABLE 1: Parameters used in the simulations and their values.

\begin{tabular}{lc}
\hline Parameter & Value \\
\hline$d_{0}$ & $100 \mathrm{~mm}$ \\
$\gamma$ & 2.2 \\
Reference tag power & $0 \mathrm{dBm}(1 \mathrm{~mW})$ \\
Signal frequency & $914 \mathrm{MHz}$ \\
\hline
\end{tabular}

TABLE 2: Average positioning error of different methods $(\sigma=3 \mathrm{~dB})$.

\begin{tabular}{lc}
\hline Method & Average error $(\mathrm{m})$ \\
\hline LANDMARC & 0.6557 \\
L-GEM & 0.5459 \\
$n$-Neurons & 1.9764 \\
Proposed method I & 0.4957 \\
Proposed method II & 0.4534 \\
\hline
\end{tabular}

The proposed methods are compared with the newly proposed RBFNN based method L-GEM [14] and the classical LANDMARC described in $[19,20]$. To show the improvement made by the fuzzy clustering radial basis center selection method, we test the same datasets with a RBFNN trained without the fuzzy clustering radial basis center selection method.

The reference tag is active tag and the power is set as $0 \mathrm{dBm}$; the signal loss from reader to reference point is set as $3 \mathrm{~dB}$. To avoid random effect, we repeat the experiment 10 times and the final result presented is the average of these experiments. Table 2 shows the experimental results when the standard deviation of fading is $3 \mathrm{~dB}$.

The LANDMARC approach $[19,20]$ is one of the most popular indoor localization technologies using active RFID tags. It is a kind of $k$-Nearest Neighbor $(k$-NN) method; the 


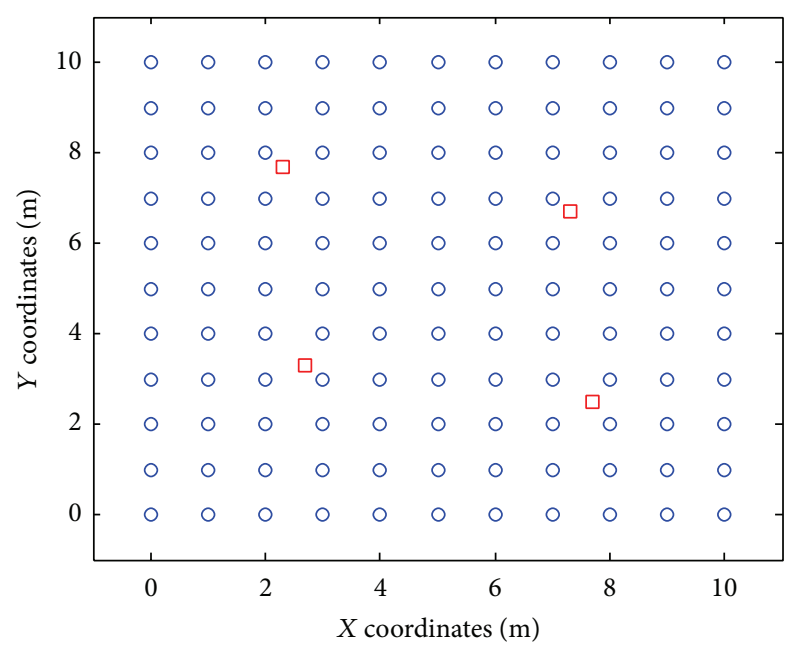

○ Reference tag

$\square$ RFID reader

FIgURE 5: The deployment of RFID readers and reference tags of Case 0.

average accuracy of LANDMARC is about 1 meter which is more precise than technologies proposed earlier. In our test, $k$ is set as 4 and the average positioning error is 0.5998 meter.

For the L-GEM approach [14], a RBFNN trained via a minimization of the localized generalization error (L-GEM) is adopted to learn the object location based on received RSSI signals. The average positioning error of this method is 0.5432 meter.

To show the improvement made by the fuzzy clustering radial basis center selection method, we test the same datasets with a RBFNN whose the number of hidden neurons is equal to the number of training samples $n$. This is a widely adopted architecture selection method of RBFNN, and we call it the " $n$-Neurons" method. Experimental result shows that the average estimation error yielded by the $n$-Neurons method is much larger than that of the fuzzy clustering based method; the average positioning error of this method is 1.9764 meter. The RBFNN trained by $n$-Neurons may be overfitting, while the RBFNN architecture selected by fuzzy clustering based method yields much better generalization capability.

In proposed method I, three separate neural networks are trained. RBFNN1 is trained by the RSSI signals and the coordinate of the reference tags, RBFNN2 is trained by the $\triangle$ RSSI signals and the coordinate of the reference tags, and the BP network is trained by the output of RBFNN1 and RBFNN2; the tutor signal is the coordinate of the reference tags.

In our test, we found that because the RSSI value of training samples is degraded by the random noise $X_{\sigma}$, the effect of using fuzzy clustering is better than the effect of using traditional hard clustering. However, we found the optimal number of clusters determined automatically via the algorithm given in Section 3.3 fluctuates in a certain range. For example, the optimal number of clusters may change in the range from 30 to 40 every time. This is likely due

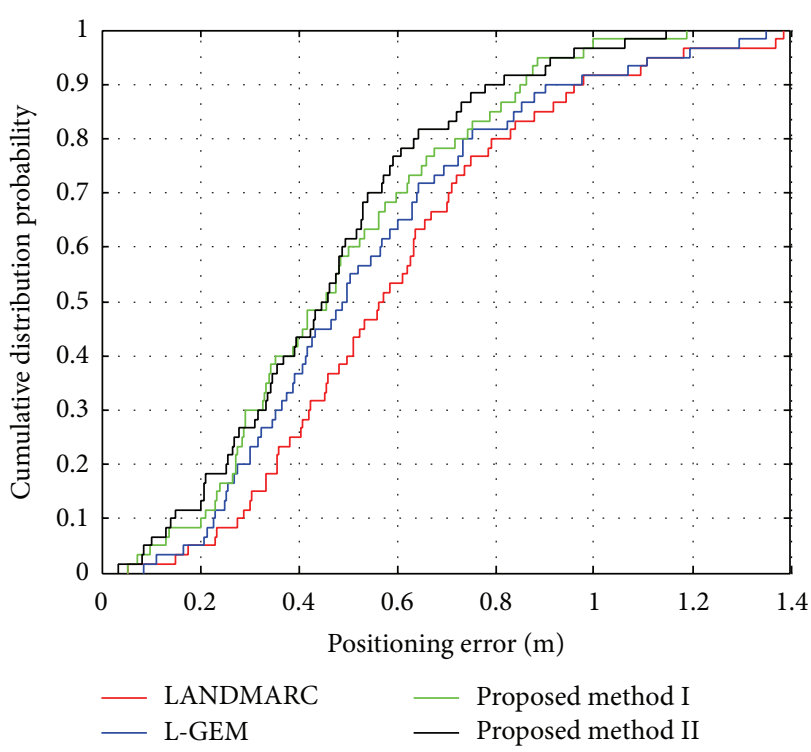

FIGURE 6: CDF of positioning error.

to the random initialization of cluster centers and degree of membership and the randomness of noise. This is also the number of neurons in the hidden layer of RBFNNs. The BP network has one hidden layer, an input layer, and an output layer, the number of hidden neurons is selected as 5. The number of neurons of input layer and output layer is 4 and 2 , respectively. So the network structure is 4-5-2. The transfer function is hyperbolic tangent sigmoid function for hidden layer and linear function for output layer. The input and output of BP neural network have been normalized.

In proposed method II, RSSI and $\Delta$ RSSI are inputs to an integrate RBF network; the number of hidden neurons is in the range of 35 to 40 via the algorithm given in Section 3.3 and is automatically determined by the algorithm. We can find from the test result that the performance of an integrate $\mathrm{RBF}$ network in method II is better than the performance of subnetwork RBFNN1 or RBFNN2 in the method I, which shows that the use of the redundant information can improve the positioning performance.

To sum up, the positioning error of the proposed methods I and II is smaller than LANDMARC's and L-GEM's, and method II is slightly better than method I.

Figure 6 shows the cumulative distribution function (CDF) of different methods' positioning error. For the LANDMARC method, $80 \%$ of tracking errors are located from $0.00 \mathrm{~m}$ to $0.80 \mathrm{~m}$ and the maximum error is $1.39 \mathrm{~m}$. For the L-GEM method, $81.7 \%$ of tracking errors are located from $0.00 \mathrm{~m}$ to $0.80 \mathrm{~m}$ and the maximum error is $1.35 \mathrm{~m}$. For the proposed method I, $85 \%$ of tracking errors are located from $0.00 \mathrm{~m}$ to $0.80 \mathrm{~m}$ and the maximum error is $1.19 \mathrm{~m}$. For the proposed method II, $90 \%$ of tracking errors are located from $0.00 \mathrm{~m}$ to $0.80 \mathrm{~m}$ and the maximum error is $1.15 \mathrm{~m}$.

The CDF curves show that the error yielded by the proposed methods is smaller than the other methods and the proposed method II is slightly better than the proposed method I. 
TABLE 3: The positioning error of different cases (m).

\begin{tabular}{|c|c|c|c|c|}
\hline \multicolumn{2}{|c|}{ Positioning error } & LANDMARC & Method I & Method II \\
\hline \multirow{4}{*}{ Case 1} & Min & 0.0959 & 0.0744 & 0.0533 \\
\hline & $\operatorname{Max}$ & 1.3731 & 1.2406 & 0.8709 \\
\hline & Mean & 0.5998 & 0.4494 & 0.3588 \\
\hline & Std. & 0.4536 & 0.3037 & 0.1877 \\
\hline \multirow{4}{*}{ Case 2} & Min & 0.1373 & 0.0918 & 0.1008 \\
\hline & $\operatorname{Max}$ & 2.7473 & 1.6833 & 1.8067 \\
\hline & Mean & 1.3528 & 0.6826 & 0.7953 \\
\hline & Std. & 0.8948 & 0.4380 & 0.6422 \\
\hline \multirow{4}{*}{ Case 3} & Min & 0.0385 & 0.1058 & 0.0334 \\
\hline & $\operatorname{Max}$ & 1.6954 & 1.6210 & 0.9608 \\
\hline & Mean & 0.7112 & 0.4631 & 0.3699 \\
\hline & Std. & 0.6801 & 0.3021 & 0.1979 \\
\hline \multirow{4}{*}{ Case 4} & Min & 0.0892 & 0.0435 & 0.0320 \\
\hline & $\operatorname{Max}$ & 1.6349 & 1.3577 & 0.8173 \\
\hline & Mean & 0.6651 & 0.4331 & 0.3143 \\
\hline & Std. & 0.4619 & 0.2749 & 0.1830 \\
\hline \multirow{4}{*}{ Case 5} & Min & 0.1402 & 0.0362 & 0.0913 \\
\hline & $\operatorname{Max}$ & 2.7319 & 1.3285 & 1.1726 \\
\hline & Mean & 1.3416 & 0.5165 & 0.4668 \\
\hline & Std. & 0.8903 & 0.2619 & 0.2503 \\
\hline
\end{tabular}

For testing the performance, we use other 5 different patterns, the details are as follows.

Case 1. The readers are deployed in the work area and the reference tag density is $0.5 \mathrm{~m}$.

Case 2. The readers are deployed in the work area and the reference tag density is $1.5 \mathrm{~m}$.

Case 3. The readers are deployed in the corner and the reference tag density is $1 \mathrm{~m}$.

Case 4. The readers are deployed in the corner and the reference tag density is $0.5 \mathrm{~m}$.

Case 5. The readers are deployed in the corner and the reference tag density is $1.5 \mathrm{~m}$.

Figure 7 shows the deployment of readers and reference tags of Case 1 . The coordinates of readers are set to the same values as abovementioned. The distances among neighboring reference tags are set to $0.5 \mathrm{~m}$ for both horizontally and vertically. Figure 8 shows the deployment of readers and reference tags of Case 5, of which the readers are deployed in the corner of the work area and the distances among neighboring reference tags are $1.5 \mathrm{~m}$ for both horizontally and vertically.

The test results are listed in Table 3, which are calculated with 10 sample runs. From Table 3, it is observed that the positioning error of the proposed algorithms is less than the positioning error of LANDMARC in several different conditions.

4.3. Computational Complexity Analysis. For method I, the neural network is composed of three subnetworks; the computational complexity of RBFNN1 is $O\left(n \cdot k_{1} \cdot l\right)$, where $n$ is

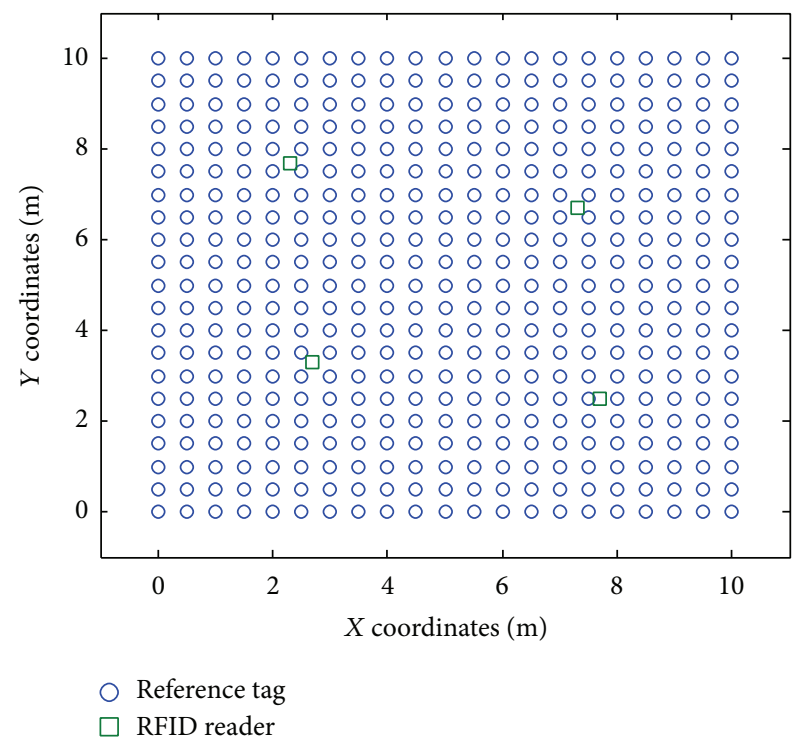

FIgURE 7: The deployment of RFID readers and reference tags of Case 1.

the number of input nodes, which is the number of RFID readers. $k_{1}$ is the number of hidden layer neurons of RBFNN1, and $l$ is the number of output nodes (i.e., $l=2$ ). Therefore, the computational complexity of RBFNN1 is $O\left(2 n \cdot k_{1}\right)$, Similarly, the computational complexity of RBFNN2 is $O\left(2(n-1) \cdot k_{2}\right)$, where $k_{2}$ is the number of hidden layer neurons of RBFNN2. The computational complexity of BP network is $O\left(2 l \cdot k_{3} \cdot l\right)=$ $\mathrm{O}\left(8 k_{3}\right)$, where $k_{3}$ is the number of hidden layer neurons of BP network. 


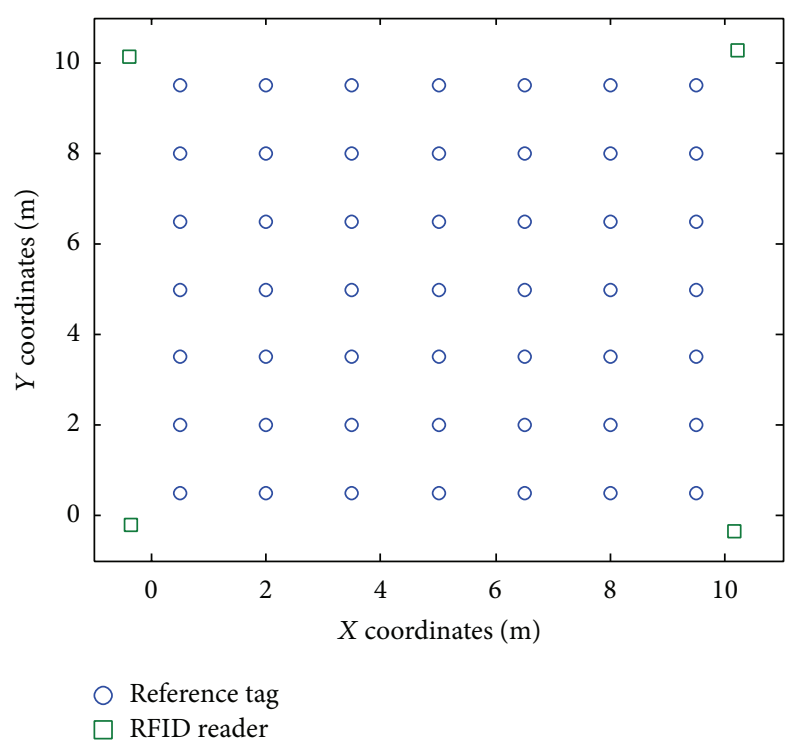

FIgURE 8: The deployment of RFID readers and reference tags of Case 5.

For method II, the neural network is a RBF neural network, whose structure is $(2 n-1)-k-l$, where $2 n-1$ is the number of input nodes, $k$ the number of hidden layer neurons, and $l$ the number of output nodes $(l=2)$. Hence, the computational complexity of method II is $O(2(2 n-1) \cdot k)$.

As a contrast, the computational complexity of LANDMARC is $O(n \cdot m)+O\left(m \cdot \log _{2} m\right) \cdot O(n \cdot m)$ corresponds to the computation of Euclidean distance and $m$ represents the number of reference tags. $O\left(m \cdot \log _{2} m\right)$ is the computational complexity of sorting the tags. Because $m$ is much greater than $n$, the computational complexity of LANDMARC is higher than that of method II.

The simulation is conducted to locate 100 tags in the work area by these algorithms. The computer is configured with Intel core I3-M370 2.4 GHz processor and 2 GB RAM. We test the positioning time of each algorithm 30 times and record their average value. The average positioning time of method I is $0.01087 \mathrm{~s}$, method II is $0.00128 \mathrm{~s}$, and LANDMARC is $0.00255 \mathrm{~s}$. The simulation result shows the running time of method II is shorter than LANDMARC, which is in line with the theoretical analysis.

\section{Conclusions}

With increasing applications of location-based Services (LBS), indoor localization technique has received much attention in recent years. Indoor localization method based on neural network has the advantages of antinoise, fast operation speed, high precision, and is a class of important indoor positioning method. However, in the existing methods, the signal inputted to neural network is measured signal itself, the differential information of measured signal is not used.
In this work, we attempted to use the difference information of the measured signal to improve the positioning performance, and take the difference information and the measured signal together as the input of the neural network. We proposed two RBFNN based indoor positioning RFID methods utilizing the RSSI signal. Method I is composed of three subnetworks, RBFNN1 learns the relationship between RSSI values and position of RFID reference tags, RBFNN2 learns the relationship between $\triangle$ RSSI values and position of RFID reference tags, and a BP neural network is trained to output the coordinate of the object. In the proposed method II, an integrate RBF neural network is trained to learn the relationship between RSSI/ $\triangle$ RSSI values and position of RFID reference tags.

In order to reduce the impact of signal fading, we improved the Gaussian filter method to process the received RSSI values. We used fuzzy clustering method to determine the centers of the radial basis function and proposed an algorithm to select the optimal number of hidden neurons for a RBFNN automatically.

The proposed methods are compared with the newly proposed RBFNN based method L-GEM and the classical LANDMARC method. Experimental results show that the proposed methods outperform the existing methods. The average estimation error of the proposed methods is no more than 0.5 meters when the standard deviation of fading is $5 \mathrm{~dB}$ in a $2 \mathrm{D}$ indoor scene.

We found that the optimal number of clusters via the algorithm given in Section 3.3 fluctuates in a certain range, but the positioning performance is basically stable. This is likely due to the random initialization of cluster centers and degree of membership and the randomness of noise. We also found that the performance of an integrate RBF network in method II is better than the performance of subnetwork RBFNN1 or RBFNN2 in the method I, which shows that the use of the redundant information can improve the positioning performance. The performance of the proposed method II is slightly better than the method I.

Through the experiment, it is proved that the proposed methods can improve the reliability and precision of the RFID indoor positioning system and are capable of indoor positioning in a noisy and multipath fading environment. In addition, the proposed methods employ an online training mechanism to improve positioning accuracy when the environment changes and relief the environment change problem in the other methods.

In the future research, we will make tentative exploration on replacing RBFNN with more efficient algorithms, such as support vector machine [21], decision tree [22], and other advanced methods.

\section{Conflict of Interests}

The authors declare that they do not have any commercial or associative interest that represents a conflict of interests in connection with the work submitted. 


\section{References}

[1] H. Liu, H. Darabi, P. Banerjee, and J. Liu, "Survey of wireless indoor positioning techniques and systems," IEEE Transactions on Systems, Man and Cybernetics C, vol. 37, no. 6, pp. 1067-1080, 2007.

[2] N. Li and B. Becerik-Gerber, "Performance-based evaluation of RFID-based indoor location sensing solutions for the built environment," Advanced Engineering Informatics, vol. 25, no. 3, pp. 535-546, 2011.

[3] B. T. Fang, "Simple solutions for hyperbolic and related position fixes," IEEE Transactions on Aerospace and Electronic Systems, vol. 26, no. 5, pp. 748-753, 1990.

[4] D. J. Torrieri, "Statistical theory of passive location systems," IEEE Transactions on Aerospace and Electronic Systems, vol. 20, no. 2, pp. 183-197, 1984.

[5] B. D. Van Veen and K. M. Buckley, "Beamforming: a versatile approach to spatial filtering," IEEE ASSP Magazine, vol. 5, no. 2, pp. 4-24, 1988.

[6] A. Teuber, B. Eissfeller, and T. Pany, "A two-stage fuzzy logic approach for wireless LAN indoor positioning," in Proceedings of the IEEE/ION Position, Location, and Navigation Symposium, pp. 730-738, April 2006.

[7] T. Ding and H. Xiao, "Chaotic time series prediction based on radial basis function network," in Proceedings of the 8th ACIS International Conference on Software Engineering, Artificial Intelligence, Networking, and Parallel/Distributed Computing (SNPD '07), vol. 1, pp. 595-599, Qingdao, China, August 2007.

[8] R. Chen and Y. Lin, "A novel method for indoor location identification," in Proceedings of the 2nd International Symposium on Aware Computing (ISAC '10), pp. 257-262, November 2010.

[9] R. J. Kuo, M. C. Shieh, J. W. Zhang, and K. Y. Chen, "The application of an artificial immune system-based back-propagation neural network with feature selection to an RFID positioning system," Robotics and Computer-Integrated Manufacturing, vol. 29, no. 6, pp. 431-438, 2013.

[10] M. Gholami, N. Cai, and R. W. Brennan, "An artificial neural network approach to the problem of wireless sensors network localization," Robotics and Computer-Integrated Manufacturing, vol. 29, no. 1, pp. 96-109, 2013.

[11] M. Kehua, C. Yaodong, and M. Xiao, "An indoor positioning technology based on GA-BP neural network," in Proceedings of the 6th International Conference on Computer Science and Education (ICCSE '11), pp. 305-309, August 2011.

[12] Y. J. Huang, C. Y. Chen, B. W. Hong, T. C. Kuo, and H. H. Yu, "Fuzzy neural network based RFID indoor location sensing technique," in Proceedings of the International Joint Conference on Neural Networks (IJCNN'10), pp. 1-5, IEEE, Barcelona, Spain, July 2010.

[13] R. J. Kuo, W. L. Tseng, F. C. Tien, and T. Warren Liao, "Application of an artificial immune system-based fuzzy neural network to a RFID-based positioning system," Computers and Industrial Engineering, vol. 63, no. 4, pp. 943-956, 2012.

[14] H. Ding, W. W. Y. Ng, P. P. K. Chan, D. Wu, X. Chen, and D. S. Yeung, "RFID indoor positioning using RBFNN with L-GEM," in Proceedings of the International Conference on Machine Learning and Cybernetics (ICMLC '10), pp. 1147-1152, Qingdao, China, July 2010.

[15] M. V. Moreno-Cano, M. A. Zamora-Izquierdo, J. Santa, and A. F. Skarmeta, "An indoor localization system based on artificial neural networks and particle filters applied to intelligent buildings," Neurocomputing, vol. 122, pp. 116-125, 2013.
[16] B. Pan, "Bias error reduction of digital image correlation using Gaussian pre-filtering," Optics and Lasers in Engineering, vol. 51, no. 10, pp. 1161-1167, 2013.

[17] S. Y. Seidel and T. S. Rappaport, "914 MHz path loss prediction models for indoor wireless communications in multifloored buildings," IEEE Transactions on Antennas and Propagation, vol. 40, no. 2, pp. 207-217, 1992.

[18] K. Z. Mao, "RBF neural network center selection based on Fisher ratio class separability measure," IEEE Transactions on Neural Networks, vol. 13, no. 5, pp. 1211-1217, 2002.

[19] L. M. Ni, Y. Liu, Y. C. Lau, and A. P. Patil, "LANDMARC: indoor location sensing using active RFID," in Proceedings of the 1st IEEE International Conference on Pervasive Computing and Communications (PerCom '03), pp. 407-415, March 2003.

[20] M. N. Lionel, Y. Liu, Y. C. Lau, and A. P. Patil, "LANDMARC: indoor location sensing using active RFID," Wireless Networks, vol. 10, no. 6, pp. 701-710, 2004.

[21] Y. Zhang, S. Wang, and Z. Dong, "Classification of Alzheimer disease based on structural magnetic resonance imaging by Kernel support vector machine decision tree," Progress in Electromagnetics Research, vol. 144, pp. 185-191, 2014.

[22] Y. Zhang, S. Wang, P. Phillips, and G. Ji, "Binary PSO with mutation operator for feature selection using decision tree applied to spam detection," Knowledge-Based Systems, vol. 64, pp. 22-31, 2014. 


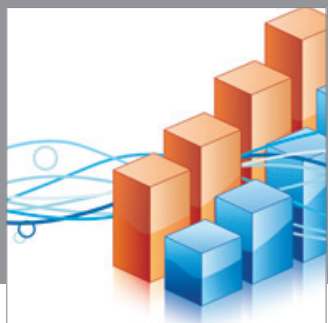

Advances in

Operations Research

mansans

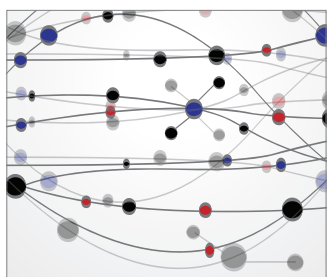

The Scientific World Journal
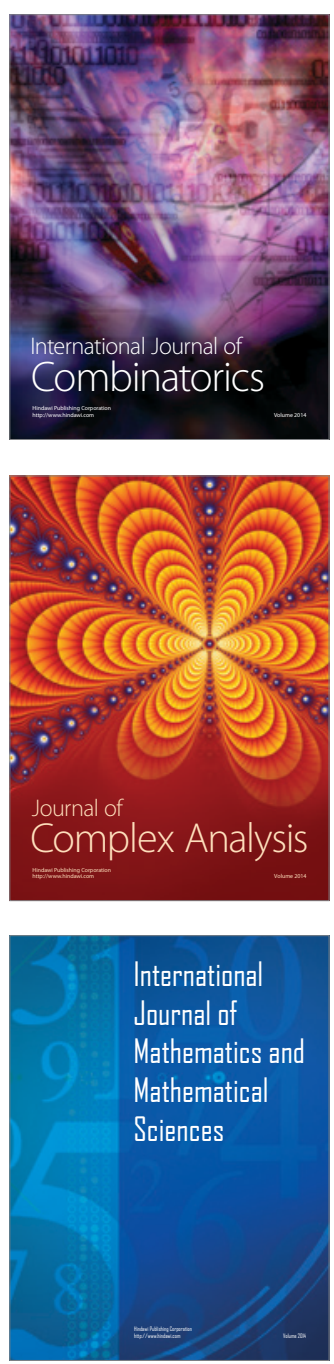
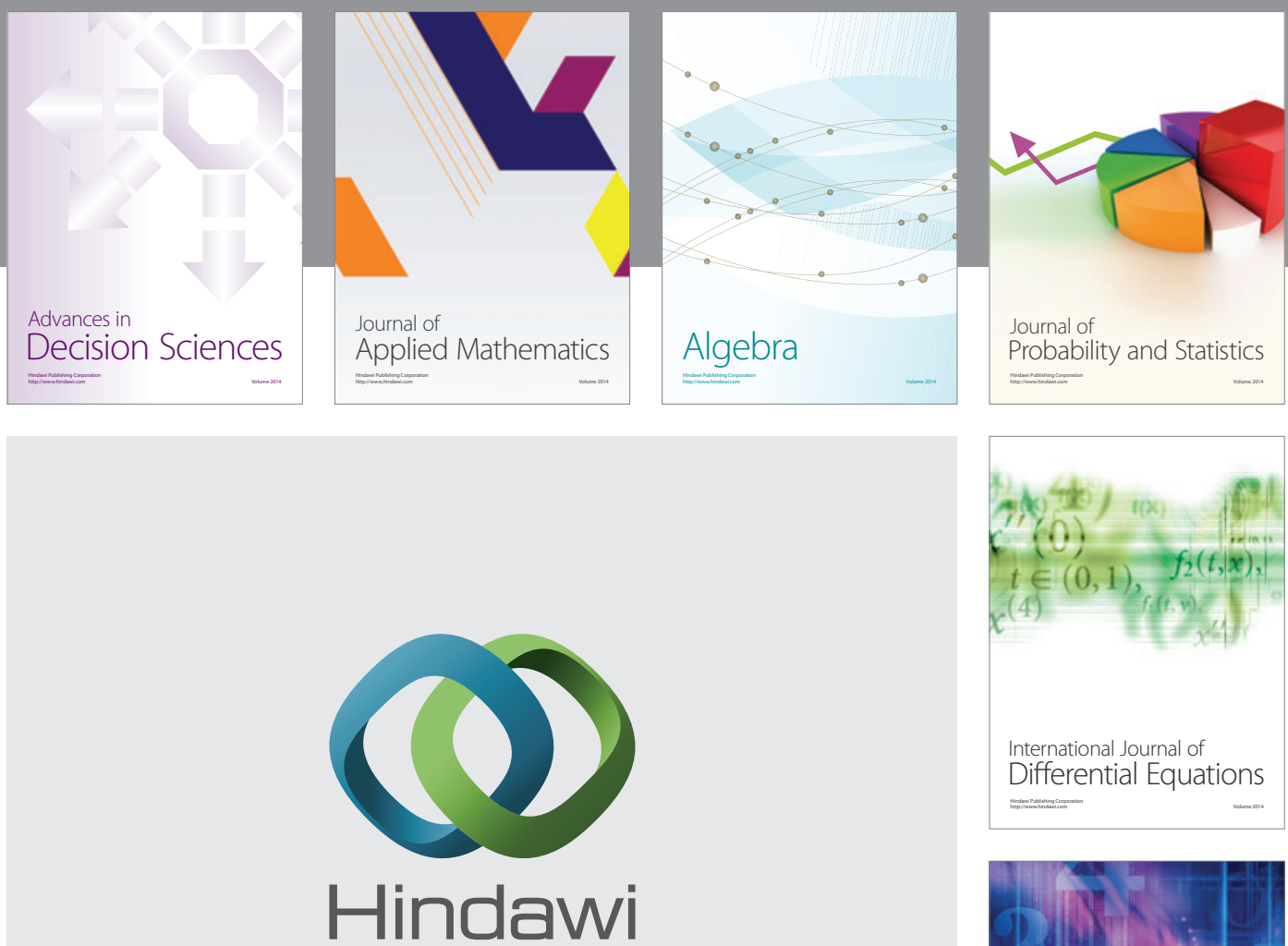

Submit your manuscripts at http://www.hindawi.com
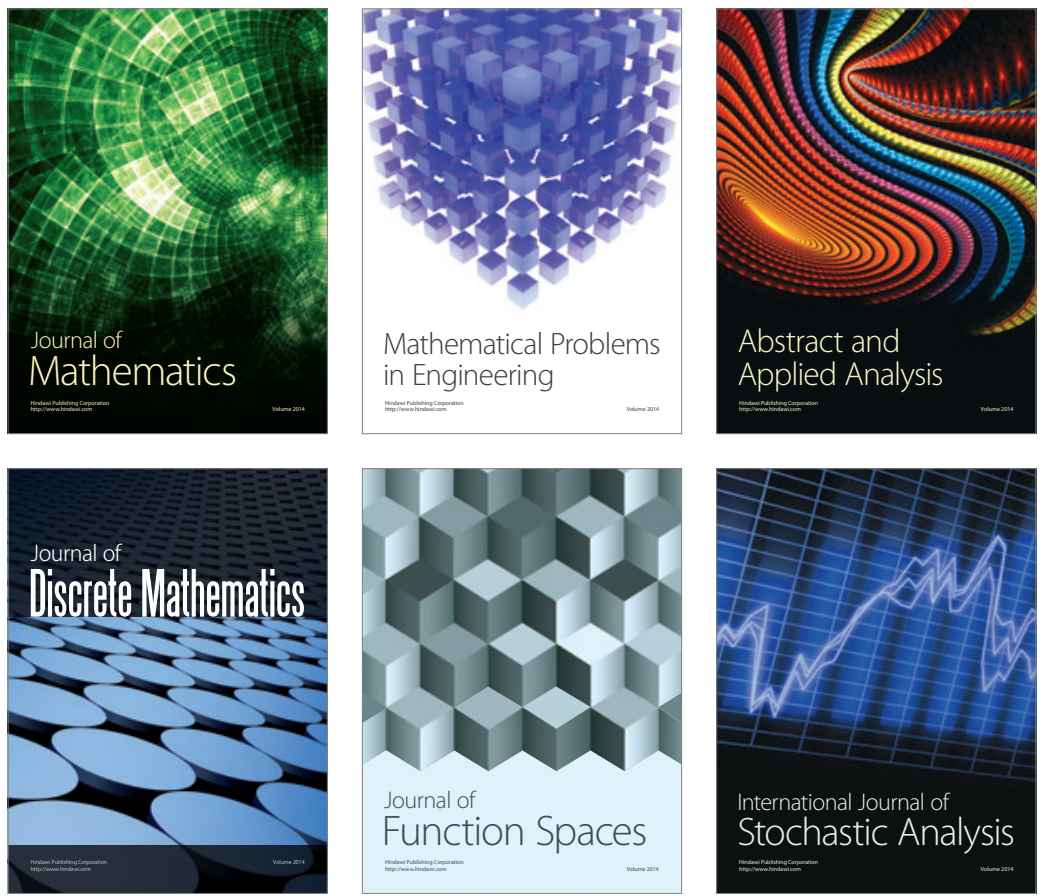

Journal of

Function Spaces

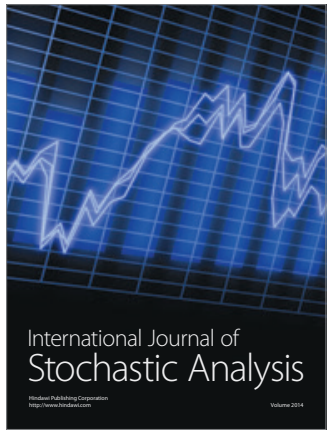

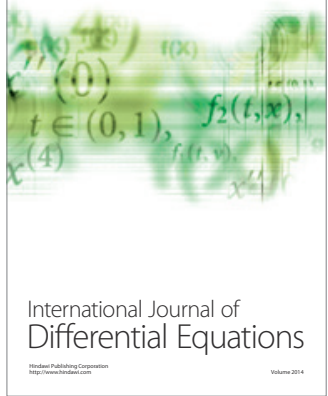
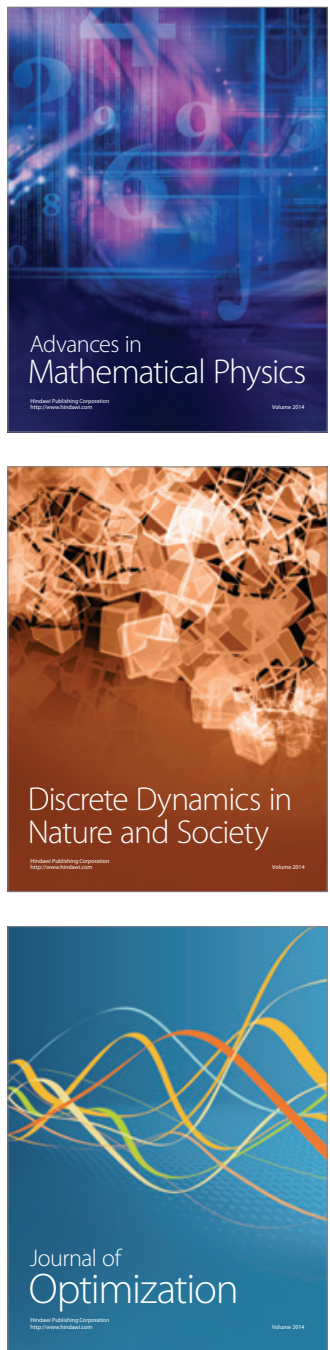